\title{
CARACTERIZACIÓN SISMOTECTÓNICA DEL NORTE DE CREVILLENTE (ALICANTE, ESPAÑA). ANÁLISIS DE LA PELIGROSIDAD Y VULNERABILIDAD DEL MUNICIPIO FRENTE AL RIESGO SÍSMICO
}

\author{
ANTONIO VICENTE GALVAÑ VICENTE \\ Graduado en Geografía y Ordenación del Territorio \\ Máster en Planificación y Gestión de Riesgos Naturales \\ Universidad de Alicante \\ avgalv3@gmail.com
}

\begin{abstract}
Resumen: A través de la presente investigación se pretende, además de llevar a cabo una caracterización sismotectónica del reborde septentrional de Crevillente (Alicante, España), analizar la peligrosidad sísmica del municipio basándose en la aplicación de una serie de métodos científicos (determinísticos, probabilísticos, EDS) y, a su vez, complementar el objetivo anterior mediante un estudio vinculado al Riesgo Sísmico del municipio, profundizando para ello en la componente de vulnerabilidad. En este último apartado, se analizan aspectos, como por ejemplo, la situación sociodemográfica actual del municipio, la vulnerabilidad sísmica de su parcelario urbano, la situación administrativo-legal del mismo en materia de riesgos o el papel que adquiere el enfoque de la gobernanza del riesgo, mostrando de esta manera la actual estructura organizativa de Crevillente, así como los déficits que imposibilitan una buena gobernabilidad o gobernanza de los riesgos naturales.
\end{abstract}

Palabras clave: Riesgo sísmico, sismicidad, vulnerabilidad, gobernanza del riesgo, Crevillente.

\begin{abstract}
Through the current investigation it is intended, in addition to carrying out a seismotectonic characterization of the northern flange of Crevillente (Alicante, Spain), to analyze the seismic hazard of the town based on the application of a series of scientific methods (deterministic, proba-
\end{abstract}

Recibido: 03-09-2020. Aceptado: 22-12-2020. 
bilistic, EDS) and, in this way, complement the previous objective through the creation of a study related to seismic risk of the town, deepening for this in the vulnerability component. Inside of the vulnerability section, features such as the socio-demographic situation of the town will be analyzed, the seismic vulnerability of its urban plot, the administrative-legal situation of the same in terms of risks or the role that acquires the risk governance approach, showing in this way not only the current Crevillente's organizational structure but also the deficits that make it impossible a good governance of natural risks.

Keywords: Seismic risk, seismicity, vulnerability, Crevillente, risk governance.

\section{Introducción}

Crevillente, como municipio situado en el sureste de la península Ibérica y, más concretamente, al sur de la provincia de Alicante, se encuentra muy próximo a una de las dos áreas de mayor actividad sísmica de España (el sureste peninsular y los Pirineos), formando parte dicho municipio del eje Cádiz-Granada-Almería-Murcia-Alicante (Canales Martínez y López Pomares, 2015). Junto a esto, el trazado próximo de fracturas cuaternarias activas hoy en día como la Falla de Crevillente o Accidente Cádiz-Alicante (Tent-Manclús, 2003) o, por ejemplo, la del Bajo Segura, Torrevieja o San Miguel de Salinas (García-Mayordomo y Martínez-Díaz, 2006) no hacen más que confirmar la importancia que adquiere el fenómeno sísmico así como la peligrosidad y vulnerabilidad a la que está sometido el municipio objeto de estudio.

Desde el punto de vista sismogenético, el municipio de Crevillente quedaría incluido dentro del vértice sismogenético número 37, correspondiente al Prebético murciano, una zona donde "la tectónica dominante la conforman fallas de dirección ENE-OSO con importante componente de desgarre y con actividad cuaternaria documentada" (Visor ZESIS, 2015). En lo relativo a la sismicidad, ésta es considerada muy abundante dentro del vértice sismogenético en cuestión, siendo la máxima magnitud registrada la del seísmo 5.4 Mw ocurrido el 28 de noviembre de 1906 en Salinas (Alicante), según lo especificado en el catálogo de cálculo utilizado por el Instituto Geográfico Nacional y la Universidad Politécnica de Madrid (IGN-UPM, 2013). Por tanto, es evidente que Crevillente queda situado en una de las zonas de la Península Ibérica que ostenta unos altos valores de peligrosidad sísmica.

De esta manera, este trabajo pretende constituirse en una herramienta con la que poder mejorar la actual situación de vulnerabilidad estructural que posee el municipio con respecto al riesgo sísmico, además de servir para concienciar a la sociedad alicantina 
sobre un peligro natural que tiende a ser infravalorado en la actualidad por la administración, ya que de los 134 municipios obligados a redactar planes de prevención contra este riesgo ninguno lo ha hecho todavía (GVA, 2020).

\section{Metodología y marco conceptual de la investigación}

Para alcanzar los objetivos definidos se ha llevado a cabo, en un primer momento, un proceso de selección de información y recopilación de material bibliográfico, tanto en formato físico (acudiendo a distintas bibliotecas de la Universidad de Alicante), como on-line, accediendo a distintos portales, repositorios o catálogos web de índole científica.

A su vez y durante la realización de la investigación, ha sido indispensable la proyección de varias salidas de campo, tanto por montaña (para conocer y entender los aspectos físicos del medio), como por el núcleo de población de Crevillente, para tomar fotografías, hacer entrevistas a diferentes actores sociales, etcétera, siendo por tanto las salidas de campo un elemento clave en este trabajo.

Otro de los aspectos determinantes a la hora de llevar a cabo el proyecto ha sido la elaboración de una amplia base cartográfica mediante técnicas SIG (Sistemas de Información Geográfica), con datos espaciales procedentes de organismos oficiales, que han sido recopilados a través de software como los programas QGIS (versión 2.14.21) y $\operatorname{ArcGIS}$ (10.2). Junto a esto, la realización de entrevistas a representantes o figuras destacadas del municipio analizado ha supuesto también (desde el punto de vista metodológico) un aspecto sumamente importante en cuanto a aporte de información, mostrándose así no solamente sus conocimientos con respecto a la amenaza sísmica sino también su opinión acerca de la actual estructura organizativa del municipio en materia de riesgos naturales.

En lo relativo al marco conceptual y a través de la transversalidad que proporciona la disciplina geográfica, se puede afirmar que éste puede perfectamente englobarse dentro del campo de estudio del análisis del riesgo, centrándose el trabajo aquí resumido sobre todo en el estudio y comprensión exhaustiva de las componentes de peligrosidad y vulnerabilidad.

\section{Resultados de la investigación}

En los próximos apartados, se especifica de forma sucinta los resultados que se han podido extraer de cada uno de los tres grandes bloques que vertebran y dan sentido a la investigación. 


\subsection{Caracterización sismotectónica de Crevillente (Alicante)}

Dentro de este primer bloque de la investigación, se tratan aspectos tan relevantes desde el punto de vista del análisis sísmico como por ejemplo la identificación gráfica, el emplazamiento, así como la contextualización sismotectónica de la zona objeto de estudio, enfatizando de esta manera en torno al trazado que describe actualmente la falla de desgarre o desplazamiento de rumbo, que supone la Falla de Crevillente o Accidente Cádiz-Alicante a su paso por los sectores más septentrionales del municipio.

Originada durante la Era Terciaria, la Falla de Crevillente es uno de los accidentes geográficos más importantes de la Península Ibérica, tanto por su antigüedad y funcionamiento en la evolución geológica de la Cordillera Bética como por las características físicas y su grado de complejidad estructural (Martínez-Díaz et al., 2002), con un recorrido que empieza en las estribaciones orientales de la provincia de Cádiz y, tras aproximadamente 200 kilómetros de longitud con orientación ENE-OSO, termina en la provincia de Alicante (Gil de la Iglesia, 2008).

En este sentido, la ocurrencia frecuente de temblores en los espacios limítrofes al trazado de la Falla de Crevillente, la presencia y relativa proximidad de otras fracturas (como la del Bajo Segura o la de San Miguel de Salinas) o las características geológicas y geomorfológicas del terreno son algunas de las múltiples evidencias físicas que corroboran el hecho de que el municipio objeto de estudio se encuentra ubicado en una de las regiones sísmicas más importantes de la Península Ibérica, como así lo refleja también el mapa de peligrosidad sísmica de España elaborado por el Instituto Geográfico Nacional en el año 2015, con valores de 0,19-0,20 g.

A raíz de la imagen anterior, se observa cómo para un periodo de retorno de 100 años, Crevillente puede recibir una intensidad sísmica máxima comprendida entre 6.5 y 7, para un periodo de retorno de 500 años dichos valores ascienden a 8-8.5 y finalmente a 8.5-9 si se contempla un escenario a 1.000 años, valores de intensidad que, en definitiva, reflejan la importancia que adquiere el fenómeno sísmico en esta zona concreta de España. Profundizando en esta cuestión, conviene recordar que gran parte de la sociedad española tiende a percibir erróneamente o malinterpretar el concepto de "periodo de retorno", presuponiendo por ejemplo que en la provincia de Alicante un evento sísmico de intensidad VII ocurre una vez cada 500 años, cuando realmente el periodo de retorno expresa que la probabilidad de que ocurra un seísmo de intensidad VII en la provincia de Alicante es de 1/500, es decir, del $0.2 \%$ anual.

A lo largo de este primer bloque de la investigación, se ha llevado a cabo también un estudio acerca de la sismicidad histórica, analizándose por un lado los principales eventos sísmicos acontecidos en épocas pasadas (utilizando para ello el catálogo de eventos sísmicos del Instituto Geográfico Nacional), proyectándose además un análisis de áreas de influencia de 10, 25 y 50 km sobre la zona de estudio, pudiéndose identificar de esta manera qué intensidades sísmicas son las más comunes en función de 


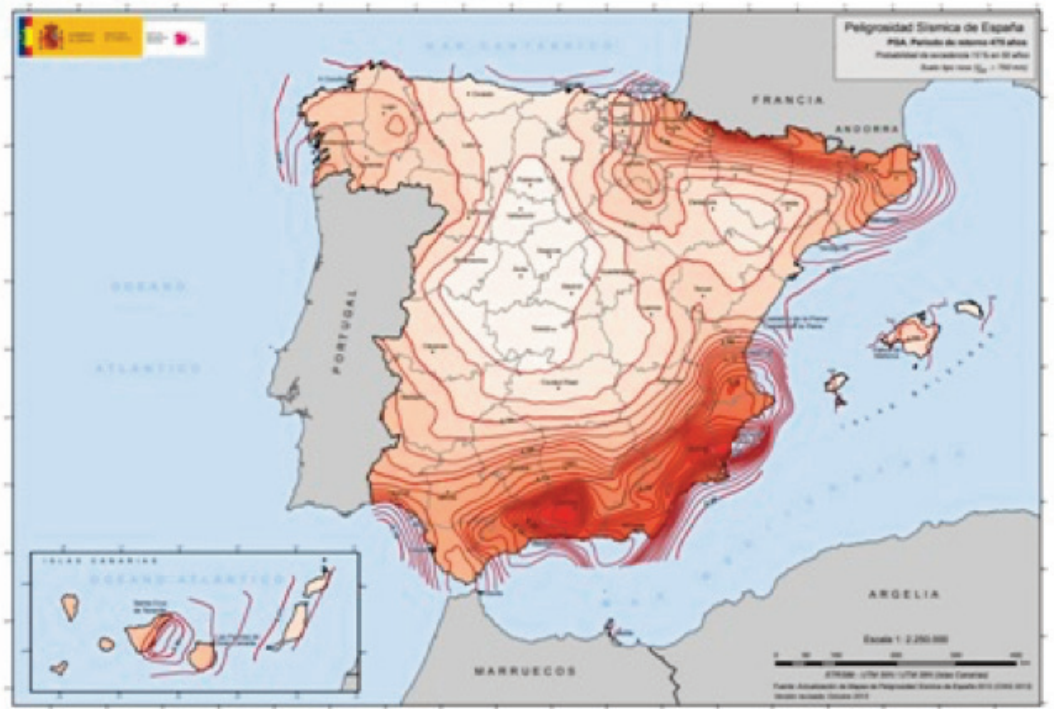

Figura 1. Mapa de peligrosidad sísmica de España (año 2015) según valores de aceleración del suelo o PGA (Peak Ground Acceleration) para T $=475$ años. Las isolíneas muestran los valores en $\mathrm{g}$, medidos según la intensidad del campo gravitatorio $\left(\mathrm{g}=9,81 \mathrm{~m} / \mathrm{s}^{2}\right)$. Fuente: Instituto Geográfico Nacional.
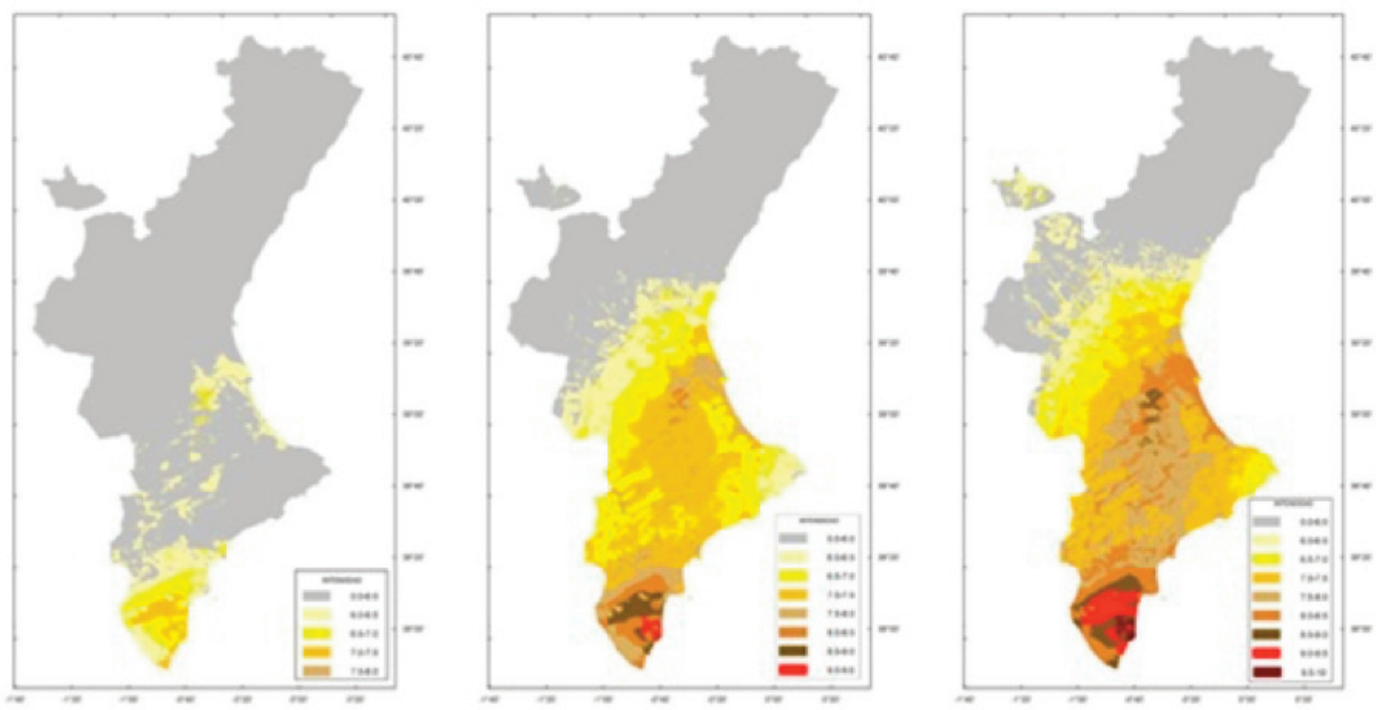

Figura 2. Intensidad sísmica esperada en la Comunidad Valenciana para un periodo de retorno (de izq. a dcha.) de 100, 500 y 1000 años.

Fuente : Unidad de Registro Sísmico de la Universidad de Alicante, 2010. 
la variable espacial y destacando además el gran peso que adquieren otros factores que, dada su naturaleza, no aparecen reflejados en la cartografía histórica elaborada, pero que aún así poseen una gran importancia en este tipo de estudios, siendo éstos la profundidad hipocentral, la distancia epicentral de los seísmos o el denominado "efecto de sitio", tratado este último con mayor profundidad en el apartado correspondiente al análisis geológico y geomorfológico de la zona de estudio.

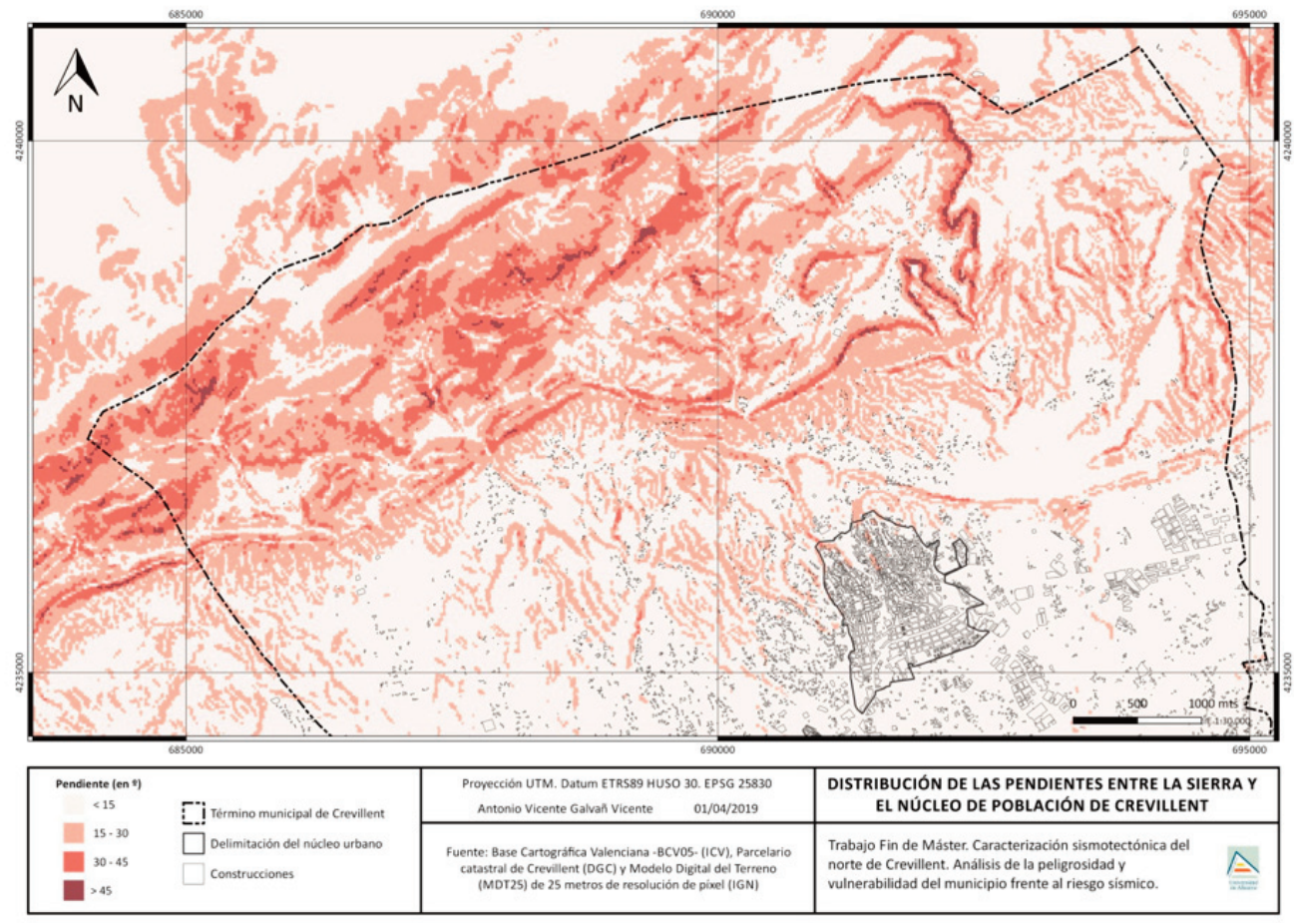

Mapa 2. Altimetría y principales desniveles orográficos (en º) en el reborde septentrional de Crevillente.

Fuente: Elaboración propia.

En cuanto a la configuración geológica de la zona de estudio, el eje montañoso de la sierra de Crevillente actuaría como línea divisoria entre los dos grandes dominios que conforman en la actualidad la zona externa de la Cordillera Bética en Alicante, esto es, el Prebético y el Subbético. Junto con el gran afloramiento jurásico calcáreo que integra los relieves más septentrionales del término municipal, encontramos litologías pertenecientes a otros periodos geológicos como el Triásico (destacando especialmente las arcillas, areniscas y yesos de tonalidades pardo-rojizas y ocres pertenecientes al Keüper, materiales con un alto contenido en hierro y localizados en torno al trazado de la Falla de Crevillente a su paso por el municipio) o los materiales miocenos y pliocenos, 
junto con los depósitos cuaternarios del Pleistoceno inferior, materiales estos últimos que configuran las morfologías más meridionales.

Desde el punto de vista geomorfológico, hay dos elementos en la zona de estudio que cobran una importancia significativa y que, a su vez, están interrelacionados entre sí, como son los fuertes contrastes altimétricos y la presencia de relieves en cuesta, representados sobre todo en los espacios más al NE del municipio. Estos condicionantes han ido forjando, desde finales del Terciario (Época Miocena-Pliocena), un relieve compuesto por geoformas de diversa índole, cuya formación y erosión posterior ha favorecido la configuración de un espacio escarpado caracterizado por el gran contraste altimétrico entre unos sectores u otros del municipio.

\subsection{Análisis de la peligrosidad sísmica del municipio}

Entendiéndose la peligrosidad sísmica como "la probabilidad de excedencia de un cierto valor de la intensidad del movimiento del suelo producido por terremotos, en un determinado emplazamiento y durante un periodo de tiempo dado" (UNDRO, 1980), se ha querido calcular para esta investigación el nivel de peligrosidad sísmica que presenta el municipio de Crevillente (Alicante), utilizando para ello una serie de metodologías de cálculo basadas en criterios determinísticos y probabilísticos. Se presentan a continuación los resultados obtenidos del estudio.

Para conocer la peligrosidad a partir del Método Determinístico no Zonificado se ha requerido el manejo del catálogo de terremotos del IGN, que ha sido sometido a un proceso de filtrado previo, posteriormente acondicionado a través de la aplicación de hojas de cálculo Excel y sobre el que finalmente se ha calculado la intensidad atenuada ${ }^{1}$ de los seísmos, en función del valor de la distancia del epicentro de los mismos a un punto de estudio en concreto, en este caso, con coordenadas $38.25^{\circ}$ latitud y $-0.81^{\circ}$ longitud. El resultado obtenido muestra cómo la intensidad sísmica esperada para Crevillente alcanza un valor de 8.72 , valor correspondiente al seísmo acontecido en Torrevieja el 21 de marzo de 1829.

Por su parte, los resultados que ofrece el Método Determinístico Zonificado son muy similares a los obtenidos con el método anterior, aunque el valor de intensidad atenuada en este último caso difiere con respecto al anterior, puesto que en su cálculo se han utilizado los vértices o fuentes sismogenéticas de la Península Ibérica definidas por el IGN. De esta manera, el punto que hemos tomado en Crevillente quedaría dentro del vértice sismogenético "NF2", por lo que el valor de la distancia sería 0 y la intensidad atenuada sería, en este caso, la misma que la del seísmo de mayor intensidad ocurrido

1. Para el cálculo de la intensidad atenuada de los seísmos se han empleado las Leyes de Atenuación del Sureste Español. (Giner Caturla, J.J., 1996). 
en dicha fuente sismogenética, ya que "la ocurrencia de un seísmo dentro de una fuente es equiprobable en el espacio y en el tiempo"2.

Se obtiene como resultado final que el valor de la intensidad sísmica atenuada según el Método Determinístico Zonificado es, para el caso de la fuente NF2 (Seísmo de Torrevieja de 1829, Alicante), de 9.5, para el vértice NF1 (Seísmo de Estubeny de 1748, Valencia), de 8.8 y, para el caso del seísmo de Vera en Almería de 1518 (vértice sismogenético NF3), de 4.9.

Tras la aplicación de esta metodología, las conclusiones son claras: la máxima intensidad sísmica esperada en Crevillente alcanza un valor de 9.5, una cifra realmente alta que, en definitiva, corrobora el hecho de que el municipio se encuentra ubicado en una región con un elevado grado de peligrosidad sísmica y por tanto debe estar preparado, tanto desde el punto de vista estructural como social, ante esta amenaza natural.

Ciñéndonos al ámbito de estudio, para el cálculo de la peligrosidad sísmica de Crevillente basado en el Método Probabilístico no Zonificado se ha optado por utilizar las funciones de distribución de valores extremos de GUMBEL I y GUMBEL III. Tras haber filtrado y homogeneizado el catálogo de eventos sísmicos de nuestra zona de estudio, haber definido la serie sísmica con los valores de intensidad del periodo temporal establecido (en este caso, 25 años), haber ajustado cada uno de los histogramas de la serie sísmica y calculado todos y cada uno de los parámetros requeridos, los resultados son los que a continuación de muestran.

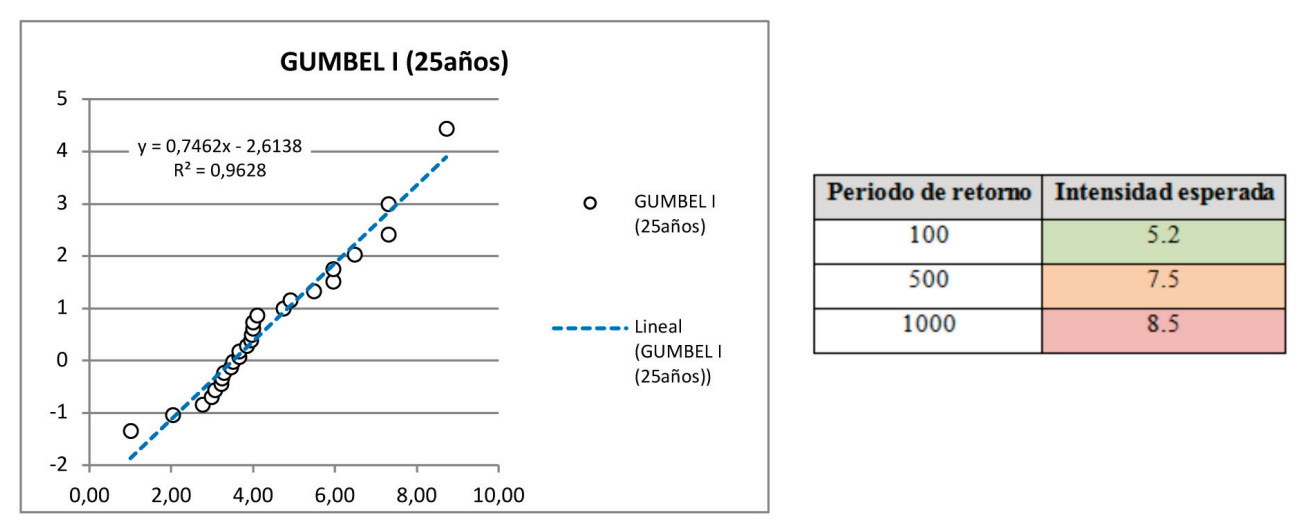

Figura 3. Gráfica de dispersión con línea tendencial de los valores extremos de la serie sísmica de 25 años según GUMBEL I. A la derecha, Intensidad sísmica esperada según GUMBEL I en función de los distintos periodos de retorno contemplados.

Fuente: Elaboración propia.

2. IGN-UPM (2013). Actualización de Mapas de Peligrosidad Sísmica de España 2012. 


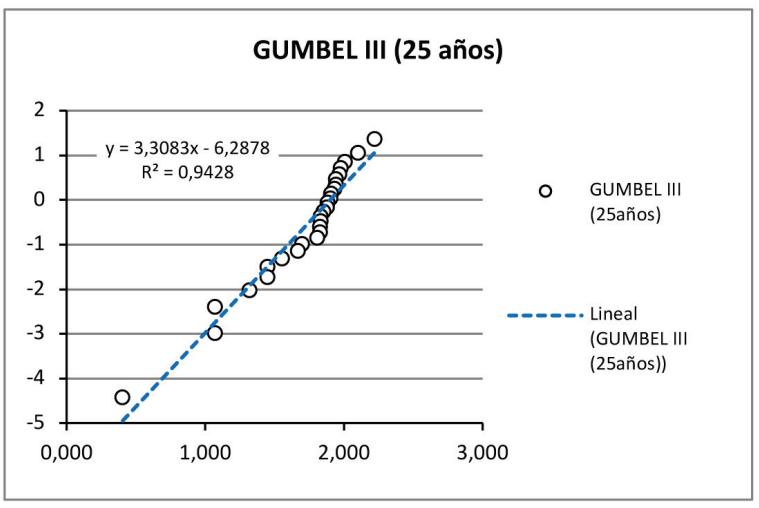

\begin{tabular}{|c|c|}
\hline Periodo de retorno & Intensidad esperada \\
\hline 100 & 5.6 \\
\hline 500 & 7.4 \\
\hline 1000 & 8.0 \\
\hline
\end{tabular}

Figura 4. Gráfica de dispersión con línea tendencial de los valores extremos de la serie sísmica de 25 años según GUMBEL III. A la derecha, Intensidad sísmica esperada según GUMBEL III en función de los distintos periodos de retorno contemplados.

Fuente: Elaboración propia.

El segundo de los métodos probabilísticos utilizados ha sido el Método Probabilístico Zonificado. Para obtener el nivel de peligrosidad sísmica al que está expuesto Crevillente según este método científico, se han empleado las fuentes sismogenéticas de la Base de Datos de ZESIS (García-Mayordomo, 2015), trabajando los datos con el software CRISIS 2015. Junto con esto, es preciso mencionar que se ha trabajado con la Ley de Atenuación de Abrahamson y Silva (1997), ya que es la ley más utilizada para zonas donde los focos sísmicos destacan por el grado de superficialidad, permitiéndonos además dicha ley estimar la aceleración sísmica en función de la magnitud. Los resultados de aceleración sísmica (en gAL $)^{3}$ para un periodo de retorno de 100 y 500 años son los que a continuación se muestran.

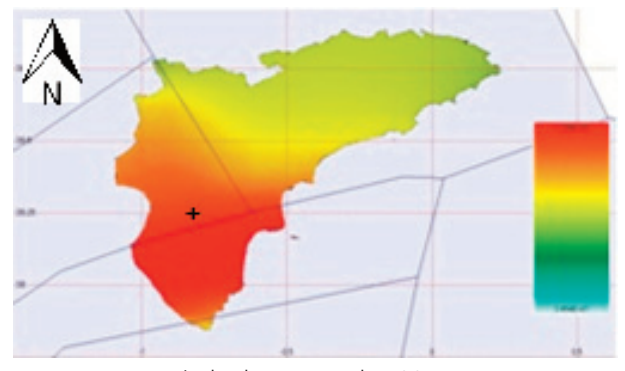

Periodo de retorno de 100 años

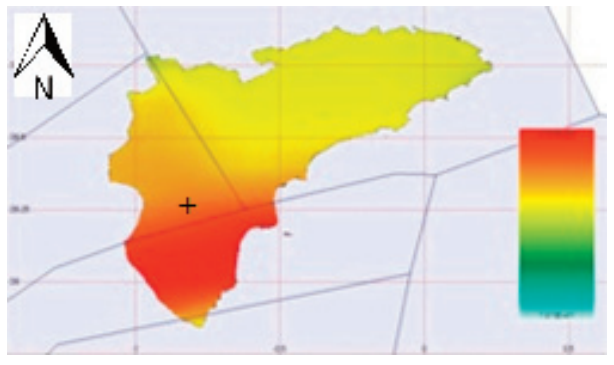

Periodo de retorno de 500 años

Figura 5. Valores de aceleración sísmica (en gAL) en la provincia de Alicante según el Método Probabilístico Zonificado. La cruz negra corresponde al núcleo de población de Crevillente.

Fuente: Elaboración propia.

3. Un gAL equivale, según lo establecido por el Sistema Internacional de Unidades (SI), a $0.01 \mathrm{metros} / \mathrm{s}^{2}$. 
Con el propósito de dar por terminado el bloque relativo al cálculo de la peligrosidad sísmica para el municipio objeto de estudio y, en función de los resultados obtenidos en los anteriores métodos (que además constatan el hecho de que Crevillente se encuentra ubicado sobre una de las regiones sísmicas más activas de España), se ha creído conveniente proyectar un Escenario de Daño Sísmico o EDS.

Para el diseño del presente EDS se ha recurrido a la aplicación Model Builder desarrollada por Medina-Cascales et al. (2017), integrada dentro del software de tratamiento de datos espaciales ArcGIS. Tras la fase de cálculo del modelo, el resultado final lo componen una serie de mapas con los que se representan los posibles daños que podría ocasionar, tanto para la población como para las infraestructuras, un seísmo de intensidad previamente definida, en nuestro caso, de grado $\mathrm{IX}^{4}$. Por otro lado, es preciso matizar que, pese a que este método nos ofrece amplios resultados en materia de vulnerabilidad, también nos proporciona cuantiosa información relacionada con la variable o el factor de peligrosidad (en este caso, a través de un mapa de distribución de ondas o intensidades sísmicas), motivo por el cual se ha optado por incluirlo dentro de este apartado. Se presentan, a continuación, los resultados obtenidos de dicho modelo.

\subsection{Estudio del Riesgo Sísmico en Crevillente (Alicante) a través de la componente de vulnerabilidad}

Tras efectuar un profundo análisis sismotectónico de la zona objeto de estudio y haber calculado el nivel de peligrosidad sísmica al que está expuesto el municipio, en este último bloque de la investigación se quiso comprobar el riesgo sísmico al que se expone Crevillente en la actualidad, por lo que para cumplir con este propósito se decidió llevar a cabo un íntegro análisis social, técnico-administrativo y estructural, todo ello mediante la componente de vulnerabilidad.

Desde el punto de vista social, dentro del apartado titulado "Breve caracterización sociodemográfica en relación con la vulnerabilidad", se efectuó, en primer lugar, un análisis censal y padronal de los efectivos demográficos del municipio (pudiendo así observar los movimientos sociodemográficos experimentados durante las últimas décadas) y, en segundo lugar, se quiso reflejar también la importancia que adquieren -desde el punto de vista de la vulnerabilidad sísmica- los movimientos o saldos migratorios así como el grado de envejecimiento o el nivel de estudios de la población.

A su vez y de manera estructural, se quiso comprobar también dentro de este apartado de la investigación el grado de vulnerabilidad que presentaba el parque de viviendas

\footnotetext{
4. Se ha seleccionado una intensidad IX (EMS) puesto que el Diario Oficial de la Generalitat Valenciana (DOGV) indica que, para un periodo de retorno de 500 años, Crevillent espera recibir dicho valor de intensidad.
} 

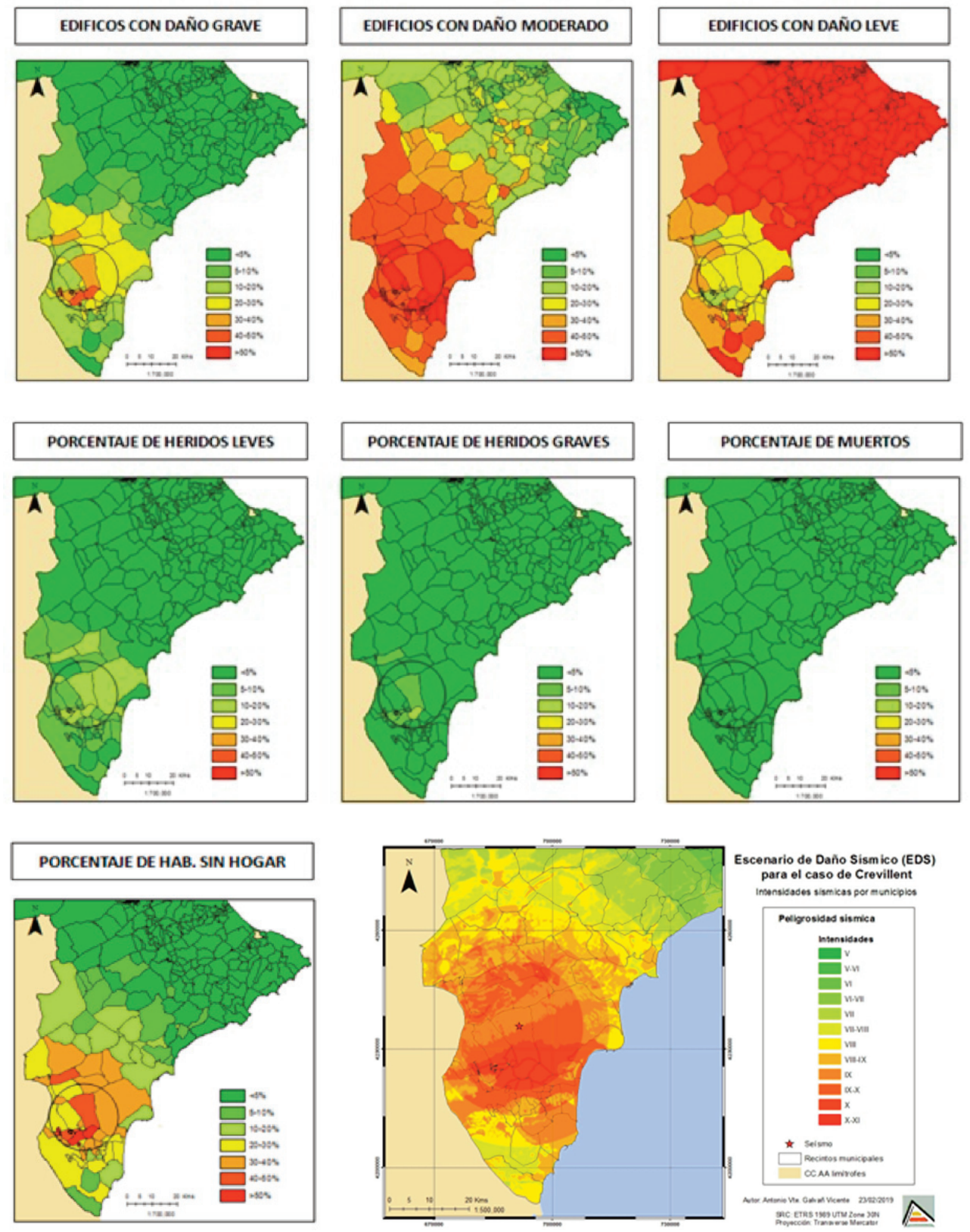

Figura 6. Síntesis de los resultados obtenidos tras la ejecución del Escenario de Daño Sísmico (EDS) en Crevillente.

Fuente: Elaboración propia. 


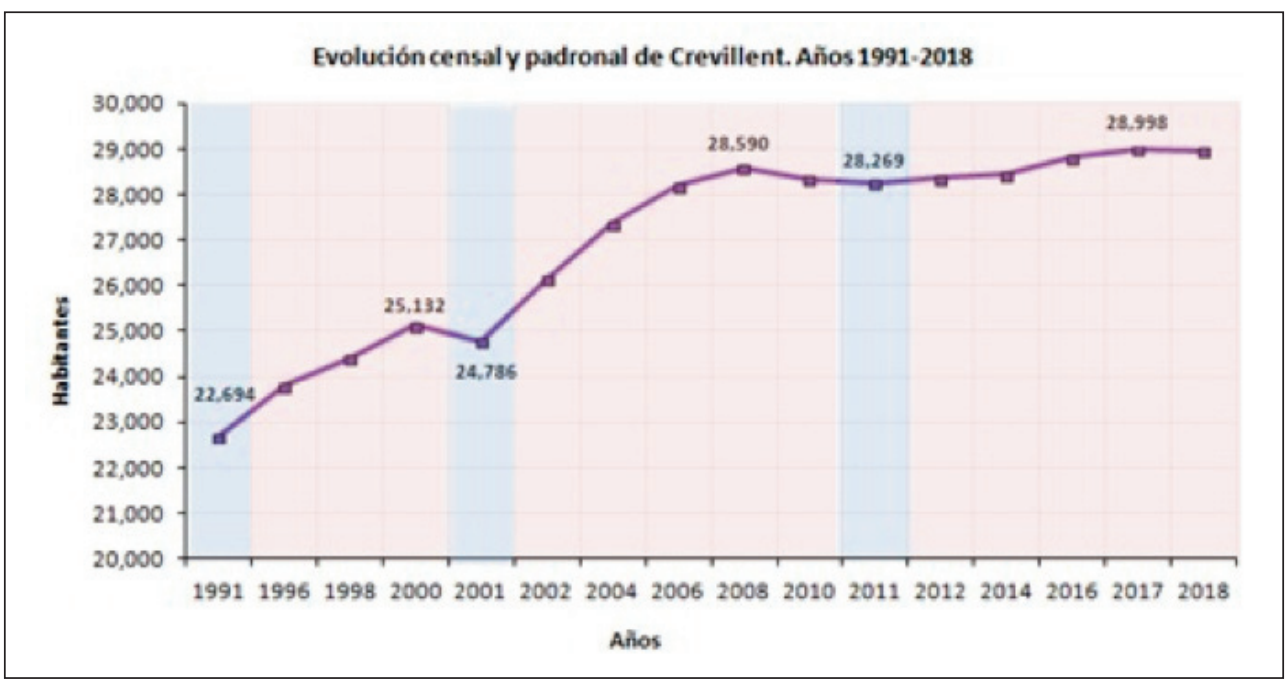

Figura 7. Evolución censal (años 1991, 2001 y 2011) y padronal (1996-2000, 2002-2010 y 2012-2018) de los efectivos demográficos del municipio de Crevillente. Periodo 1991-2018.

Fuente: Ajuntament de Crevillente e Instituto Nacional de Estadística. Elaboración propia.
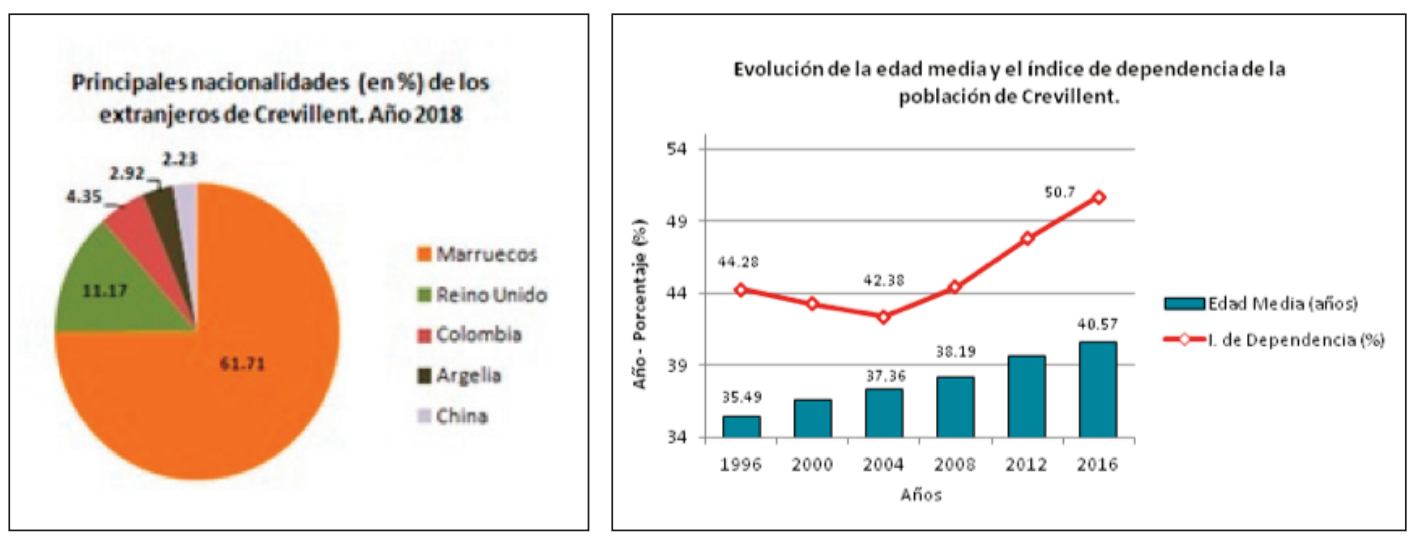

A la izquierda, Figura 8. Principales nacionalidades (en \%) de los extranjeros residentes en Crevillente. A la derecha, Figura 9. Evolución de la edad media y del índice de dependencia de la población del municipio. 
que conforma el núcleo de población principal de Crevillente con respecto a la amenaza sísmica. Para materializar este propósito, se trabajó con el parámetro relativo a la antigüedad del parcelario urbano, tomando para ello los datos recogidos en la Sede Electrónica de la Dirección General de Catastro5. Es preciso destacar que antes de iniciar el proceso de asignación del año de cada parcela, fue necesario acometer una corrección del plano catastral dadas las notables deficiencias (polígonos shapefile duplicados o mal ubicados), que presentaban los datos en la mayoría de las secciones censales que conformaban los 4 distritos en los que se estructura el núcleo de población.

El resultado final fueron un total de 18 composiciones (una por cada sección censal), las cuales incluyen un mapa, un gráfico circular y un diagrama de barras en los que poder comprobar y analizar de forma exhaustiva el grado de vulnerabilidad de las edificaciones que integran cada una de ellas. Pueden consultarse íntegramente en el Anexo II de la presente investigación.

Una vez contextualizadas todas las viviendas del núcleo de población de Crevillente en función de su antigüedad, se clasificaron todas ellas (un total de 10085) según clases de vulnerabilidad específicas. Tras este proceso, se cruzaron los datos obtenidos con unas matrices de probabilidad de daño (en este caso, las planteadas por Chávez en el año 2003), obteniendo de esta manera la cuantía total de viviendas que, ante un evento sísmico de relativa importancia, sufrirían daño leve (2459), moderado (5300) y grave (2326).

Por otro lado, dentro de este último bloque de la investigación se quiso profundizar acerca de la actual situación administrativo-legal del municipio en materia de riesgos naturales, haciendo especial hincapié en el riesgo sísmico. En este sentido y para el caso de estudio que nos ocupa, los dos documentos que se consolidan como los más relevantes dentro de la normativa de ámbito local en materia de riesgos son, en primer lugar, el Plan General de Ordenación Urbana o PGOU (aprobado en 2011) y, por otro lado, la redacción y consiguiente aprobación (en agosto de 2018) del Plan Territorial frente a Emergencias de Crevillente. Tras haberlos revisado detenidamente, se pueden extraer una serie de conclusiones, que se especifican a continuación:

- En lo que respecta a la actualización del Plan General de Ordenación Urbana efectuada en 2016, únicamente se hace mención (y de forma realmente escueta) al posible riesgo de inundación existente en las zonas más meridionales del término municipal (San Felipe Neri, El Realengo, entre otras pedanías), como consecuencia de la posible ruptura de la presa en forma de escollera que delimita un sector del pantano de Crevillente. Otro de los aspectos a destacar es la inexistencia, en todo el documento, de recursos cartográficos que evidencien los riesgos naturales a los que está expuesto el municipio.

5. Sede Electrónica del Catastro. Gobierno de España. Enlace web: https://www.sedecatastro.gob.es/ 

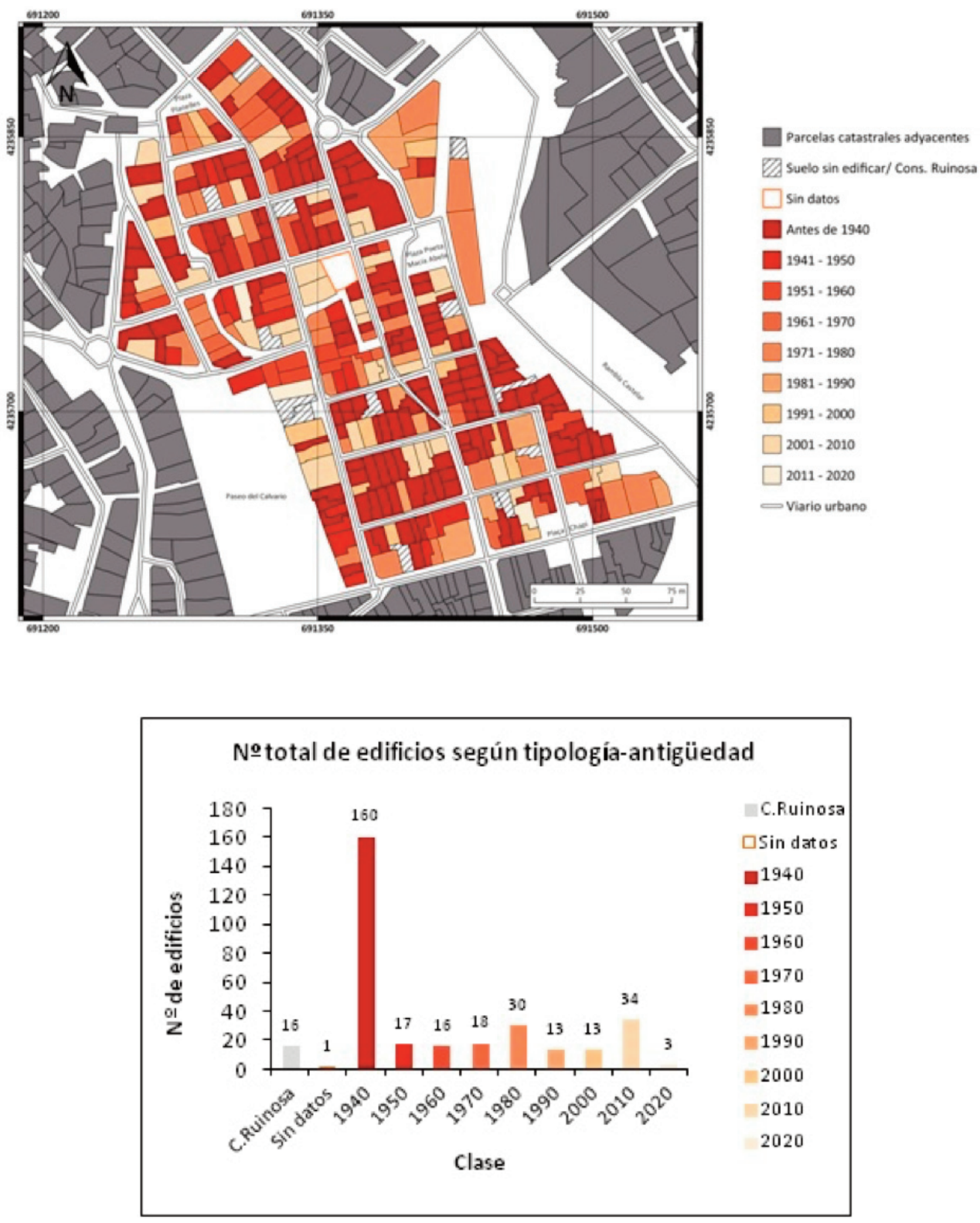

Figura 10. Mapa y gráfico de barras adjunto que muestran el grado de vulnerabilidad sísmica de los inmuebles incluidos dentro de la sección censal

01 del Distrito 3 del municipio de Crevillente (Alicante).

Fuente: Elaboración propia. 


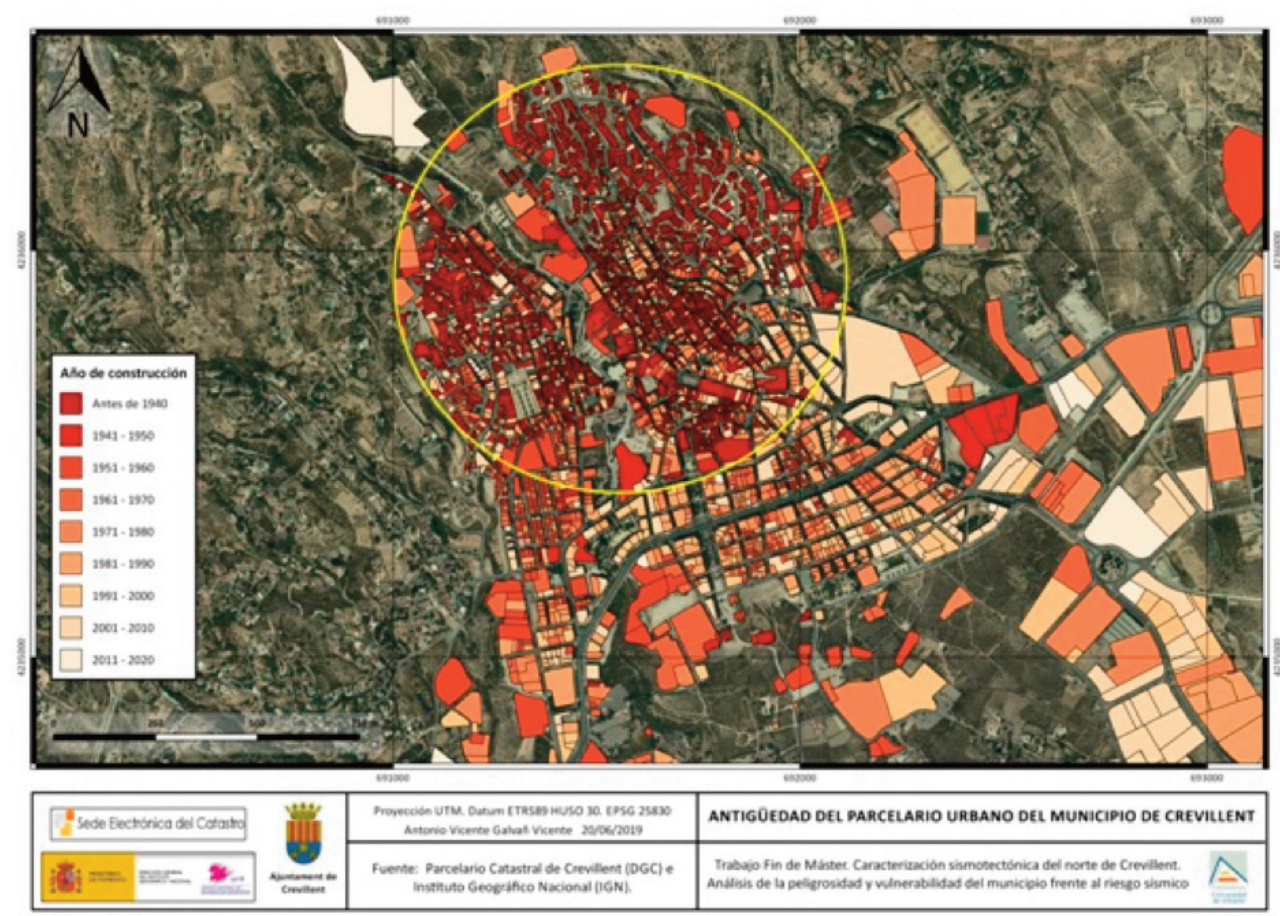

Mapa 3. Vulnerabilidad sísmica del parcelario urbano del municipio de Crevillente. Fuente: Elaboración propia.

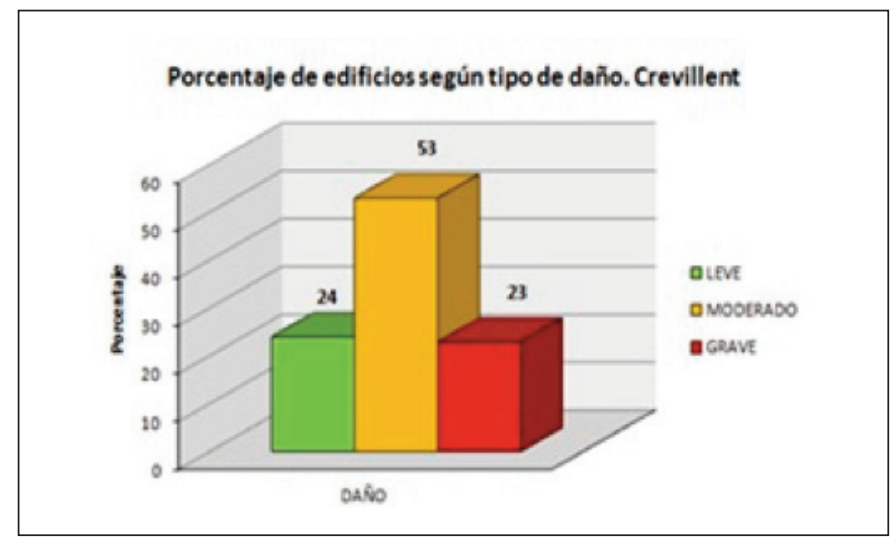

Figura 11. Porcentaje de edificios afectados en Crevillente según el tipo de daño recibido.

Fuente: Elaboración propia. 
Tabla 1. Clasificación de los inmuebles de Crevillente a raíz del daño sufrido ante un posible seísmo en función de la máxima intensidad sísmica esperada para un periodo de retorno de 500 años, según el Diari Oficial de la Generalitat Valenciana (DOGV) ${ }^{6}$

\begin{tabular}{|c|c|c|c|c|}
\hline \multicolumn{1}{|c|}{ codIN } & Municipio & \multicolumn{3}{c|}{ Daño } \\
\hline 03059 & Crevillent & LEVE & MODERADO & GRAVE \\
\hline \multicolumn{2}{|c|}{ TOTAL } & 2459 & 5300 & 2326 \\
\hline
\end{tabular}

Fuente: Elaboración propia.

- El segundo de los documentos que conforma la normativa de ámbito local frente a los riesgos naturales con incidencia en el municipio es el conocido como Plan Territorial frente a Emergencias de Crevillente o PTEM (2018), elaborado siguiendo las directrices que establece el Plan Territorial de Emergencias de la Comunidad Valenciana. Conviene destacar también (al igual que sucedía con el PGOU) la poca información existente en dicha normativa local acerca del riesgo sísmico aunque, sin lugar a dudas, lo más sorprendente de este segundo documento normativo es la ausencia total de planes específicos o de actuación municipal (PAM) frente a emergencias.

En virtud de los resultados obtenidos en cada uno de los apartados anteriores de la investigación y tras haber efectuado un análisis exhaustivo de la estructura normativa en materia de riesgos naturales existente en Crevillente, se pudo identificar (mediante la deconstrucción del concepto de Gobernabilidad o Gobernanza del Riesgo) una serie de stakeholders o actores sobre los que recae un mayor peso a la hora de gestionar y hacer frente a los riesgos que repercuten sobre el municipio, comprendiendo además de qué manera entienden ellos el riesgo, sobre todo el sísmico. Se han detectado también, a través de la reunión con los propios actores entrevistados, una serie de barreras o déficits cuya existencia ha determinado y condicionado la situación de vulnerabilidad actual del municipio con respecto a la amenaza sísmica.

Tal y como se desprende de la Figura 12, la estructura organizativa de Crevillente en relación con el riesgo sísmico giraría en torno a 4 entes o bloques principales, siendo éstos la administración local (ayuntamiento), el sector educativo (colegios o institutos del municipio), el sector empresarial (empresas o industrias) y la sociedad civil, integrada por entidades o asociaciones como Protección Civil y Cruz Roja, entre otras. Bajo el papel que ejercen cada uno de estos cuatro actores identificados se encuentra la so-

6. Según lo especificado en el DECRETO 44/2011, de 29 de abril, por el que se aprueba el Plan Especial frente al Riesgo Sísmico en la Comunitat Valenciana. [2011/5012]. 
Tabla 2. Riesgos naturales analizados y cartografía de riesgos incluida en el Plan General de Ordenación Urbana de Crevillente del año 2011. Consultado en Olcina-Cantos et al. (2018)

\begin{tabular}{|l|l|l|}
\hline \multirow{2}{*}{ Principales riesgos } & \multicolumn{2}{|c|}{ Municipio de Crevillente } \\
\cline { 2 - 3 } & $\begin{array}{c}\text { Riesgos tratados en } \\
\text { el último PGOU }\end{array}$ & $\begin{array}{c}\text { Riesgos tratados en } \\
\text { el último PGOU }\end{array}$ \\
\hline Riesgo de inundación & & \\
\hline Riesgo sísmico & & \\
\hline Riesgo de erosión & & \\
\hline Riesgo de deslizamientos & & \\
\hline Vulnerabilidad de acuíferos & & \\
\hline Riesgo de incendios forestales & & \\
\hline Riesgo de intrusión marina & & \\
\hline Riesgo de desprendimientos & & \\
\hline Riesgo de colapso & & \\
\hline Riesgo de subsidencia & & \\
\hline Riesgos asociados a procesos kársticos & & \\
\hline
\end{tabular}

Fuente: Elaboración propia.

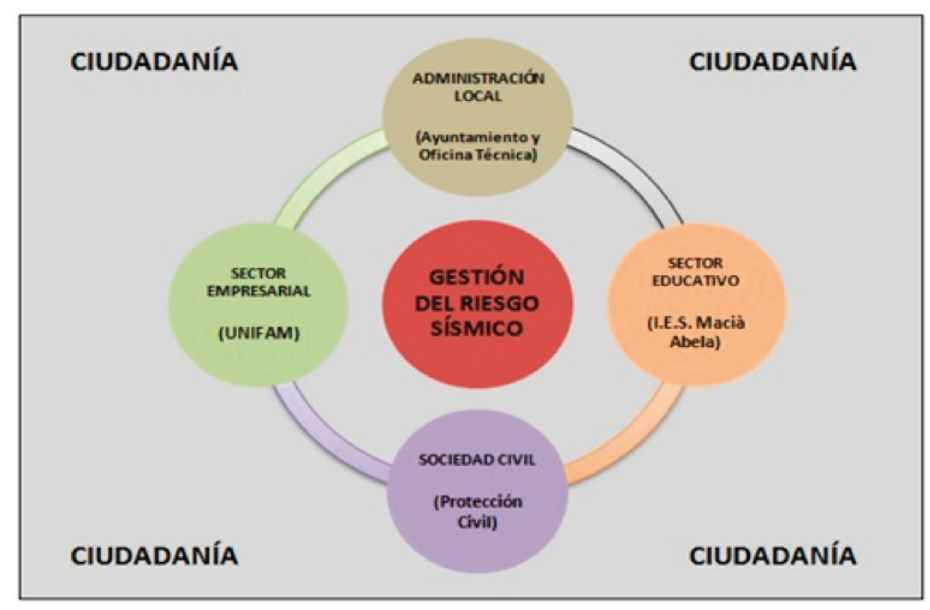

Figura 12. Stakeholders identificados en el municipio de Crevillente (Alicante) en relación con la gestión del riesgo sísmico.

Fuente: Elaboración propia. 
ciedad o ciudadanía de Crevillente, stakeholder principal sobre el que se sustenta todo el enfoque que supone la gobernanza del riesgo, ya que en él repercuten todas las medidas, acciones o estrategias implantadas por los demás actores.

Ante la obtención de estos resultados y tras la realización de las respectivas entrevistas, lo cierto es que durante los últimos años no ha habido una gestión totalmente eficaz a nivel municipal o, mejor dicho, no se ha diseñado una correcta gobernanza del riesgo sísmico en Crevillente. En este sentido, se hace patente una falta de coordinación o entendimiento entre los diferentes stakeholders implicados en todo este proceso, descoordinación que influye además en la configuración de una situación de desinformación hacía la ciudadanía. Ante esta situación, la falta de aprobación de planes de actuación contra emergencias (PAM's) así como la escasa difusión de los planes ya redactados conlleva un aumento de la vulnerabilidad de la población, grupo social que, fruto de la desinformación, tiende a desestimar la importancia que adquieren los riesgos naturales en el municipio.

\section{Conclusiones}

A lo largo de los tres bloques que componen la presente investigación, se han podido obtener una serie de conclusiones:

- Por un lado, se ha profundizado en el conocimiento geológico y geomorfológico de la zona de estudio, identificando y describiendo además el comportamiento de la Falla de Crevillente o Accidente Cádiz-Alicante a su paso por los espacios más septentrionales del municipio, esto es, su propio eje montañoso.

- Se ha establecido una relación causa-efecto entre la actividad sísmica producida por la Falla de Crevillente y la disposición o configuración que presentan actualmente los relieves más próximos al trazado de ésta, analizando la sismicidad histórica y corroborando que la actividad tectónica acontecida desde el Messiniense, hace \pm 7 millones de años, ha ido alterando sustancialmente la disposición de los materiales, los cuales estuvieron sometidos en un primer momento a unos grandes esfuerzos tectónicos, flexionándose y levantándose violentamente durante la orogenia alpina, y siguen estando hoy en día sometidos a esas fuerzas neotectónicas, aunque de manera mucho más calmada, experimentando modificaciones u alteraciones prácticamente imperceptibles, del orden de los 6 milímetros/año (Geolodía 18 Alicante, 2018).

- Por su parte, se ha podido comprobar también como los materiales plío-cuaternarios son los que presentan una amplificación de onda sísmica mayor, debido en parte a sus características físicas. El núcleo de población de Crevillente se encuentra 
ubicado sobre este tipo de materiales, factor éste que debe sin duda ser tenido en cuenta en futuros trabajos de determinación de peligrosidad o vulnerabilidad.

- Tras analizar la vulnerabilidad sísmica del parcelario urbano del municipio, se ha podido comprobar la antigüedad del mismo, sobre todo de los inmuebles que integran los distritos censales más septentrionales del parque de viviendas, esto es, los distritos 3 y 4 . En este sentido, sería conveniente actuar y tratar de hacer menos vulnerables los espacios incluidos dentro del área que delimita el círculo amarillo del Mapa 3 adjunto en este artículo, proyectando acciones de rehabilitación, modificaciones estructurales (económicamente viables) o plasmando medidas no estructurales, es decir, informar y tratar de dotar de conocimientos a la población para que sepa actuar en caso de seísmo.

- Con respecto a la vulnerabilidad, se manifiesta además la imperiosa necesidad de redactar y aprobar cuanto antes todos los Planes de Actuación Municipal contra riesgos con afección en el municipio todavía pendientes, desde aquellos que se postulan como absolutamente necesarios (como el sísmico, el de incendios forestales o el de sequías) hasta aquellos con una repercusión o impacto sobre el municipio menor, como por ejemplo el PAM contra el riesgo de nevadas o contra el riesgo de deslizamientos. Es preciso destacar también que, tras la redacción y aprobación de cualquier normativa de ámbito local en materia de riesgos naturales, la difusión entre la ciudadanía (charlas, reuniones, medios de comunicación local, etc.), así como el sometimiento a revisiones y actualizaciones periódicas de esos documentos, deberá constituirse como un aspecto ineludible y de primer orden. De nada sirve cumplir o disponer de todos los planes de prevención o actuación frente a riesgos si la ciudadanía no es partícipe o no es informada de ellos.

- Por otro lado, resulta fundamental también que todos los actores sociales que conforman la actual sociedad o estructura organizativa de Crevillente (Administración, Sector Educativo, Sector Empresarial, Colectivos o Asociaciones Civiles y demás actores implicados) participen más activamente, colaboren y establezcan una mayor coordinación entre sí, favoreciendo de esta manera la detección y subsanación de déficits u obstáculos que puedan surgir de todo este proceso y posibilitar así una correcta gobernanza de los riesgos naturales en el municipio.

- No se trata de alarmar a la sociedad sino todo lo contrario, el objetivo primordial radica en que la ciudadanía disponga de unos conocimientos básicos acerca del fenómeno sísmico, que esté sensibilizada al respecto y que sepa actuar ante una situación de emergencia. En este sentido, la implementación, tanto de medidas no estructurales (educación, transmisión de información, aprobación de todos los planes de actuación y respuesta necesarios, coordinación eficaz entre los stakeholders), como estructurales (modificación o acondicionamiento de la trama urbana de las ciudades, inspección periódica de edificios, Inspecciones Técnicas de 
Edificaciones, etc.) se postulan como propuestas o medidas encaminadas a reducir los niveles de vulnerabilidad o de exposición de la población frente a posibles amenazas, consiguiendo en definitiva ciudades más resilientes.

\section{Bibliografía}

Abrahamson, N. y Silva, W. (1997). Empirical Response Spectral Attenuation Relations for Shallow Crustal Earthquakes. Seismological Research Letters, 68 (1), pp. 94-127. DOI: https:// doi.org/10.1785/gssrl.68.1.94

Ajuntament de Crevillent (2018). Plan Teritorial Municipal frente a Emergencias. Crevillent, 81.

Canales, G. y López, A. (2015). Riesgos naturales en la Huerta de Orihuela. En: Ferrández, T. y Diz, E. (Cood.). Historia Natural de la Huerta de Oribuela (pp. 251-283). Ayuntamiento de Orihuela.

Chávez, J. (1998). Evaluación de la vulnerabilidad y el riesgo sísmico a escala regional: Aplicación a Cataluña. Tesis doctoral. Universidad Politécnica de Cataluña. Barcelona, pp. 323.

DECRETO 44/2011, de 29 de abril, por el que se aprueba el Plan Especial frente al Riesgo Sísmico en la Comunitat Valenciana. [2011/5012], 154.

García-Mayordomo, J. y Martínez-Díaz, J.J. (2006). Caracterización sísmica del Anticlinorio del Bajo Segura (Alicante): Fallas del Bajo Segura, Torrevieja y San Miguel de Salinas. Geogaceta, 40, pp. 19-22.

García-Mayordomo, J. (2015). Creación de un modelo de zonas sismogénicas para el cálculo del mapa de peligrosidad sísmica de España. Madrid: Instituto Geológico y Minero de España.

Gil de la Iglesia, A. (2008). Estudio de fallas activas en la Cordillera Bética Oriental entre 3-o-Oo

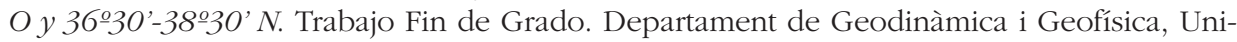
versitat de Barcelona, pp. 63.

Giner, J.J., Molina, S. y Jáuregui, P.J. (1996). Peligrosidad Sísmica en la Comunidad Valenciana. En: I Congreso Iberoamericano sobre técnicas aplicadas a la gestión de emergencias para la reducción de desastres naturales. Universidad Politécnica de Valencia, I, pp. 341-357.

GVA (2020). Listado de Municipios de la Comunitat Valenciana con obligación de tener un Plan de actuación Sísmica. Agencia Valenciana de Seguridad y Respuesta a las Emergencias.

IGN-UPM (2013). Actualización de Mapas de Peligrosidad Sísmica de España 2012. Madrid: Centro Nacional de Información Geográfica.

Martínez-Díaz, J.J., Riego, A., Louis, L., Capote del Villar, R., Hernández, J.L., Carreño, E. y Tsige, M. (2002). Caracterización geológica y sismotectónica del terremoto de Mula (febrero de 1999, Mb: 4,8) mediante la utilización de datos geológicos, sismológicos y de interferometría de RADAR (INSAR). Boletín Geológico y Minero, 113 (1), pp. 23-33.

Medina-Cascales, I., Soler-Llorens, J.L. y Giner, J.J. (2017). Cálculo de Escenarios de Daños Sísmicos en la Comunidad Valenciana utilizando Model Builder (ArcGIS). Geogaceta, 62, pp. 67-70. 
Olcina-Cantos, J., Morote, Á.F. y Hernández, M. (2018). Evaluación de los riesgos naturales en las políticas de ordenación urbana de los municipios de la provincia de Alicante. Legislación y cartografía de riesgo. Cuadernos Geográficos, 57 (3), pp. 152-176. DOI: http://dx.doi.org/ 10.30827/cuadgeo.v57i3.6390

Tent-Manclús, J.E. (2003). Estructura y estratigrafía de las sierras de Crevillente, Abanilla y Algayat: su relación con la falla de Crevillente. Tesis doctoral. Universidad de Alicante. Alicante, pp. 1008.

UNDRO (1980). Natural Disasters and Vulnerability Analysis. Office of the United Nations Disaster Relief Co-ordinator. Génova: UNDRO.

VVAA (2018). Geolodía 18 Alicante. Crevillent. 13 de mayo de 2018. Alicante: Universidad de Alicante. 